\title{
Errors in pigeons' memory for number of events
}

\author{
ANGELO SANTI and CHRIS HOPE \\ Wilfrid Laurier University, Waterloo, Ontario, Canada
}

\begin{abstract}
In Experiment 1, pigeons were trained in a within-subjects design to discriminate sequences of light flashes (illumination of the feeder) that varied in number, but not in time (2f/4sec and $8 \mathrm{f} / 4 \mathrm{sec})$, and in time, but not in number (4f/2sec and $4 \mathrm{f} / 8 \mathrm{sec})$. Number samples required a response to one of two comparison dimensions (either color or line), whereas time samples required a response to the remaining comparison dimension. Delay testing revealed a significant choose-small bias following number samples and a significant choose-long bias following time samples. In Experiment 2, testing confirmed that in the absence of a sample, there was a bias to respond small to the number comparisons and long to the time comparisons. Additional tests indicated that the birds were discriminating time samples on the basis of the number of light flashes occurring during the last few seconds of the time samples, rather than on the basis of the total duration of the flash sequence. Consequently, the choose-long bias observed for time samples during delay testing was really a choose-small bias. In Experiment 3, the birds received baseline training with a 5-sec delay and were subsequently tested at shorter and longer delays. A choose-large bias occurred at delays shorter than the baseline training delay, whereas a choose-small bias was again observed at delays longer than the baseline delay. These findings provide additional empirical support for the conceptualizing of memory for number and time in terms of a common mechanism.
\end{abstract}

A number of studies have employed a delayed matchingto-sample procedure to investigate memory for event duration and memory for number of events in pigeons. In a typical experiment on memory for event duration, trials begin with the presentation of a continuous signal (e.g., the overhead houselight) for either a short (e.g., $2 \mathrm{sec}$ ) or a long (e.g., $8 \mathrm{sec}$ ) duration. Termination of the sample is followed immediately by the presentation of two comparison stimuli. A peck to one comparison stimulus (e.g., red) is reinforced if the sample duration was short, whereas a peck to the other comparison stimulus (e.g., green) is reinforced if the sample duration was long. Following acquisition, memory for time is assessed by inserting delay intervals of varying length between the termination of the sample and the onset of the comparisons. Previous research has shown that at delays longer than baseline training, pigeons continue to respond with high accuracy following the short-duration sample, whereas accuracy of responding to the long-duration sample drops to well below 50\% correct (Fetterman, 1995; Gaitan \& Wixted, 2000; Grant, 1993; Grant \& Spetch, 1993, 1994; Kelly \& Spetch, 2000; Kraemer, Mazmanian, \& Roberts, 1985; Santi, Bridson, \& Ducharme, 1993; Santi, Ducharme, \& Bridson, 1992; Sherburne, Zentall, \& Kaiser, 1998;

This research was supported by Grant OGPOOD6378 from the Natural Sciences and Engineering Research Council of Canada to A.S. The authors thank Marion Corrick for her technical assistance and James Coyle, whose previous research on this topic in our laboratory greatly facilitated the execution of this study. Correspondence concerning this article should be addressed to A. Santi, Department of Psychology, Wilfrid Laurier University, Waterloo, ON, N2L 3C5 Canada (e-mail: asanti@wlu.ca).
Spetch, 1987; Spetch \& Rusak, 1989, 1992; Spetch \& Wilkie, 1983). This result is commonly known as the choose-short effect, because pigeons show a bias to peck the comparison stimulus that is correct for the shortduration sample as the delay interval is extended beyond that used in training.

Similar experiments have been conducted to assess memory for number of events in pigeons. In this case, the sample stimulus consists of a small number or a large number of equally spaced light flashes, each lasting $200 \mathrm{msec}$. For example, the small sample would consist of two flashes of light in $4 \mathrm{sec}(2 \mathrm{f} / 4 \mathrm{sec})$, whereas the large sample would consist of eight flashes of light in $4 \mathrm{sec}$ $(8 \mathrm{f} / 4 \mathrm{sec})$. A peck to one comparison stimulus (e.g., red) is reinforced if the sample number was small, whereas a peck to the other comparison stimulus (e.g., green) is reinforced if the sample number was large. Roberts, Macuda, and Brodbeck (1995) used this type of procedure and found that, during delay testing, pigeons continued to respond with high accuracy following the samples consisting of a small number of light flashes $(2 \mathrm{f} / 4 \mathrm{sec})$, whereas the accuracy of responding following the large number $(8 \mathrm{f} / 4 \mathrm{sec})$ dropped to well below $50 \%$ correct. Fetterman (2000) reported a similar finding with rates of stimulus change (slow vs. fast) serving as sample stimuli. Delay tests indicated a bias to respond to the comparison alternative associated with the slow sample. Fetterman (2000) hypothesized that rate discriminations were based on the number of sample events (light flashes) and that the choose-slow bias represented an instance of a choosesmall effect. Thus, memory for number reveals a choosesmall effect, just as memory for time reveals a chooseshort effect. 
These findings, in conjunction with other research on the simultaneous processing of time and number information by rats (Meck \& Church, 1983) and pigeons (Roberts \& Mitchell, 1994), have supported the view that the processing of temporal and numerical information is accomplished by a common mechanism. Different theoretical approaches have articulated the nature of this common mechanism in different ways. The dual-mode version of scalar timing theory advanced by Meck and Church proposed that the same pacemaker-accumulator system processes both time and number. Broadbent, Church, Meck, and Rakitin (1993) have proposed a connectionist model of timing and counting, whereas Staddon and Higa (1999) have advanced a multiple time scale model in which both time and number are stored as values of a decaying memory established either by a continuous signal (duration) or by a sequence of events (number).

The objective of the present study was to provide additional empirical support for the conceptualizing of memory for number and time in terms of a common mechanism, rather than to differentiate among the different theoretical approaches to this mechanism. However, in the present paper, the theoretical framework of the mode control model is adopted, because it was the earliest theoretical position advanced and also because it is the perspective that has been dominant in theoretical interpretations of previous research on memory for time and number (see Roberts, 1998; Roberts et al., 1995; Spetch \& Wilkie, 1983).

The mode control model advanced by Meck and Church (1983) suggested that the processing of time and number is accomplished by the accumulation of pulses in separate accumulators. According to this model, a pacemaker generates a constant stream of pulses, which are gated by external signals into accumulators. Operation of the gating mechanism in different modes accounts for temporal and numerical processing of stimulus events. In the run mode, pulses are gated to a temporal accumulator whenever a stimulus event begins, and they only stop being transmitted when the stimulus event terminates. In the event mode, pulses are briefly gated to a numerical accumulator at the beginning of each stimulus event. Consequently, if the stimulus event consists of a sequence of light flashes, then at the end of the sequence, the temporal and numerical accumulators will have recorded a different total number of pulses, and these totals would correspond to the total duration of the sequence and the total number of stimulus events in the sequence. This model can account for data showing that both rats (Meck \& Church, 1983 ) and pigeons (Roberts \& Mitchell, 1994) can process time and number information simultaneously.

Roberts and Mitchell (1994) extended the mode control model by showing that separate working memory stores for time and number were required. They accomplished this by training pigeons with sequences of light flashes for which different responses (peck left or peck right) were required with respect to the time or the num- ber attribute of each sequence. Postsequence cues signaled whether the correct response for a sequence was based on its temporal attribute or its numerical attribute. Pigeons were able to successfully use the postsequence cues to accurately respond on the basis of their memory for the ambiguous sequences. The demonstration that temporal and numerical information can be selectively retrieved from working memory was explained within the mode control model by assuming that time and number accumulators transfer their totals to separate stores in working memory.

If temporal and numerical processing is accomplished by the same theoretical mechanism, variables that affect memory for event duration should have the same effect on memory for number, and vice versa. Although much of the previous research on memory for event duration involved training at a 0 -sec baseline delay, a few studies have trained pigeons at a longer baseline delay (e.g., 5 or $10 \mathrm{sec}$ ) and then tested at delays shorter or longer than the baseline delay. In contrast to the choose-short effect observed at test delays longer than the baseline delay, testing at delays shorter than the baseline delay results in a choose-long effect (Spetch, 1987; Spetch \& Rusak, 1989). This choose-long effect presumably occurs because memory for an event duration at a short test delay would appear larger than the foreshortened values occurring at the longer baseline training delay. If time and number processing involves a common mechanism, one would expect tests of memory for number at delays shorter than the training delay to reveal a choose-large effect.

The following experiments had two objectives: (1) to replicate the findings of Roberts et al. (1995) on memory for number and extend them to a within-subjects design and (2) to demonstrate that response biases in memory for number show the same sensitivity to baseline training delay as is the case in memory for time.

\section{EXPERIMENT 1}

Experiment 1 was conducted to replicate the findings of Roberts et al. (1995) and extend them to a withinsubjects design. Pigeons were trained to discriminate sequences of light flashes (illumination of the feeder) that varied in number, but not in time $(2 \mathrm{f} / 4 \mathrm{sec}$ and $8 \mathrm{f} / 4 \mathrm{sec})$, and in time, but not in number $(4 \mathrm{f} / 2 \mathrm{sec}$ and $4 \mathrm{f} / 8 \mathrm{sec})$. For half of the birds, number samples required a response to color comparison stimuli (red and green), whereas time samples required a response to line orientation comparison stimuli (vertical and horizontal lines). This was reversed for the remaining birds. Training was conducted with a 0 -sec baseline delay, and testing was conducted at delays of $0,5,10,15$, and $20 \mathrm{sec}$. On the basis of the findings of Roberts et al. (1995), it was expected that delay tests following the number samples would exhibit a significant choose-small bias. That is, as the delay was lengthened the pigeons were expected to respond with high accuracy following the samples consisting of a small number 
of flashes $(2 \mathrm{f} / 4 \mathrm{sec})$, whereas their accuracy following the samples consisting of a large number of flashes $(8 \mathrm{f} / 4 \mathrm{sec})$ was expected to drop to $50 \%$ correct or lower.

In the study conducted by Roberts et al. (1995), a separate group of pigeons (the time group) had been trained to discriminate sequences of light flashes that varied in time, but not in number $(4 \mathrm{f} / 2 \mathrm{sec}$ and $4 \mathrm{f} / 8 \mathrm{sec})$. During delay testing, the time group exhibited an unexpected choose-long effect, as opposed to the expected chooseshort effect that normally occurs when pigeons time a continuous signal. Transfer tests supported the hypothesis that the pigeons in this group were using the number of light flashes at the end of a sample sequence as a discriminative cue. For example, the long sample $(4 \mathrm{f} / 8 \mathrm{sec})$ was processed as if it had consisted of a small number of events (e.g., $1 \mathrm{f}$ in the last $2 \mathrm{sec}$ ), whereas the short sample $(4 \mathrm{f} / 2 \mathrm{sec})$ was processed as if it had consisted of a large number of events ( $4 \mathrm{f}$ in the last $2 \mathrm{sec}$ ). Consequently, the time samples were processed by the birds as if they were number samples. Roberts et al. (1995) concluded that the choose-long effect in the time group was actually a choose-small effect. On the basis of these results, it was expected that delay tests following the time samples in the present study would also exhibit a significant choose-long effect (functionally, a choose-small effect).

\section{Method}

Subjects. The subjects were 12 experimentally naive adult Silver King pigeons. They were maintained at approximately $80 \%$ of their free-feeding weight, with constant access to water and grit. Postsession feedings of Purina Pigeon Chow were provided to maintain their target weights. The pigeons were individually housed in a room maintained at approximately $22^{\circ} \mathrm{C}$. The colony room was illuminated on a 12:12-h light:dark cycle by fluorescent light turned on at 6:00 a.m. each day. Testing was conducted 6 days a week between 9:00 a.m. and 3:00 p.m.

Apparatus. Four Coulbourn modular operant test cages (Model E10-10), each housed within isolation cubicles (Model 10-20), were used. Each cubicle utilized baffled air intake exhaust systems and ventilation fans. Each test cage contained three horizontally aligned, translucent plastic keys positioned approximately at a pigeon's standing sight line. Behind each key was a projector that displayed red, green, or white onto a frosted rear projection screen (Coulbourn Model E21-18). Vertical and horizontal lines were presented as white bars with a black background. Directly below the center key there was a $5.7 \times 5 \mathrm{~cm}$ opening that, during reinforcement, provided access to a hopper containing mixed grain. Within the opening was a lamp (Coulbourn Model E14-10 with bulb S11819X) that was illuminated only during reinforcement. Located $6.5 \mathrm{~cm}$ above the center key was a houselight that directed light upward to reflect light from the top of the cage (Coulbourn Model 14-10). The organization of all experimental events and the recording of response choices were performed by a microcomputer system located in the same room.

Procedure. All the birds were trained to eat mixed grain from the illuminated food hopper. After hopper training, the pigeons were autoshaped to peck at red, green, vertical line, or horizontal line stimuli randomly presented on either the left or the right side keys. Any bird that was not reliably pecking the illuminated keys by the end of the sixth session was manually shaped. Training began once all the birds were reliably pecking the side keys on at least $85 \%$ of the trials.

Each trial commenced with a sample presentation in which the hopper light flashed on and off throughout a sequence. Each se- quence began and ended with a $200-\mathrm{msec}$ light flash. The remainder of the 200-msec flashes occurred at equally spaced time intervals between the beginning and the ending flash. The number samples consisted of two flashes in $4 \mathrm{sec}(2 \mathrm{f} / 4 \mathrm{sec})$ or eight flashes in $4 \mathrm{sec}(8 \mathrm{f} / 4 \mathrm{sec})$. The time samples consisted of four flashes in $2 \mathrm{sec}$ $(4 \mathrm{f} / 2 \mathrm{sec})$ or four flashes in $8 \mathrm{sec}(4 \mathrm{f} / 8 \mathrm{sec})$. The order of presentation of the sample stimuli was randomized independently for each bird. For 6 birds, color comparisons were presented after the number samples and line comparisons were presented after the time samples. For these birds, red was correct following the small $(2 \mathrm{f} / 4 \mathrm{sec})$ sample, green was correct following the large $(8 \mathrm{f} / 4 \mathrm{sec})$ sample, vertical was correct following the short $(4 \mathrm{f} / 2 \mathrm{sec})$ sample, and horizontal was correct after the long $(4 \mathrm{f} / 8 \mathrm{sec})$ sample. For the remaining birds, line comparisons were presented after the number samples, and color comparisons were presented after the time samples. For these birds, vertical was correct following the small sample, horizontal was correct following the large sample, red was correct following the short sample, and green was correct after the long sample. For all the birds, a single peck to the comparison stimuli turned them off and, if correct, permitted 4-sec access to mixed grain from the food hopper. Incorrect responses to the comparison stimuli resulted in a 4-sec blackout, followed immediately by the presentation of the same sample and comparison stimulus configuration. A correct response on a correction trial produced 4-sec access to mixed grain, although only the choice response on the initial (noncorrection) trial was used to calculate matching accuracy. Within each block of 8 trials, all combinations of the four sample stimuli with the comparison stimuli, counterbalanced on the left and right side keys, occurred once. The order of presentation was randomized individually for each bird. The intertrial interval, spent in darkness, was $15 \mathrm{sec}$. All the sessions ended upon completion of 80 trials. Training continued for 25 sessions. Over the last 5 sessions of 0 -sec training, accuracy was at or above $80 \%$ correct. On the last day of training, the birds averaged $86 \%$ correct on the number trials and $85 \%$ correct on the time trials.

Delay testing was conducted for 15 sessions of 160 trials each. Within each session, 24 trials for each of the four samples (small, large, short, and long) occurred at the 0 -sec baseline delay, and 4 trials for each sample occurred at each of the other delays $(5,10,15$, and $20 \mathrm{sec}$ ). This distribution of delays $(60 \%$ baseline delay, $40 \%$ other delays) was used so that the reference memory of temporal and numerical samples and their association with the comparison stimuli established during 0 -sec baseline delay training would remain stable during testing (Spetch \& Wilkie, 1983). During delay testing, the correction procedure remained in effect only for 0-sec-delay trials. All other parameters remained the same as those described for training.

In all the statistical analyses reported in this article, the rejection region was $p<.05$.

\section{Results and Discussion}

The mean percentage of correct responding during delay test sessions is shown in Figure 1. The data for trials in which number samples were presented are shown in the top of the figure. For the number samples, a choosesmall bias was observed. Accuracy dropped much more on the large-sample trials than on the small-sample trials as the delay interval increased. For the number samples, significant effects of sample type $[F(1,11)=76.29]$ and delay interval $[F(3,44)=181.38]$ and a sample type $\times$ delay interval interaction $[F(3,44)=57.19]$ were obtained. At the 0 -sec delay, accuracy was slightly higher on the large-sample trials than on the small-sample trials $[F(1,11)=6.20]$. However, at all delays greater than $0 \mathrm{sec}$, accuracy was much higher on small-sample trials 

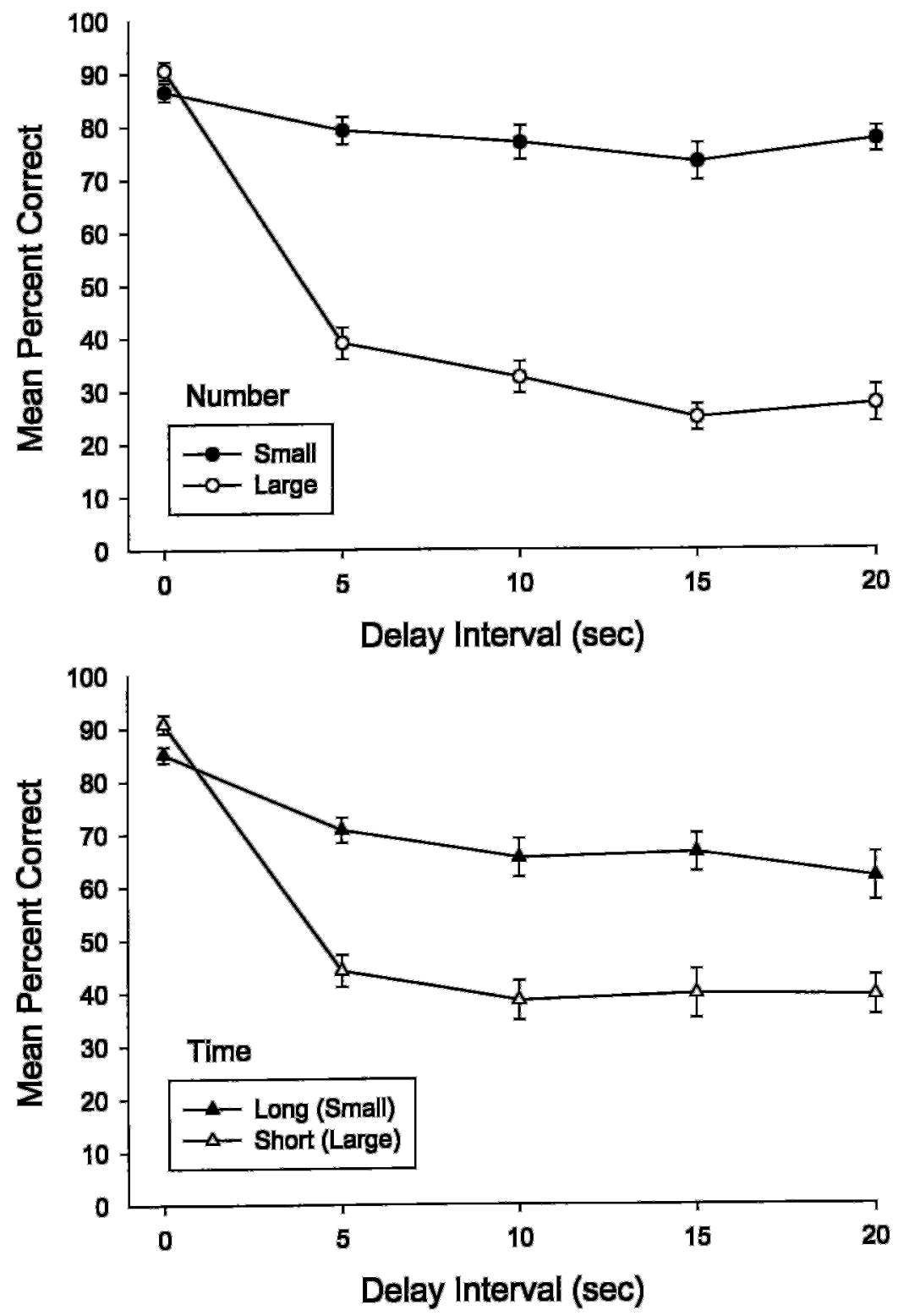

Figure 1. The mean percentage of correct responding during delay testing sessions in Experiment 1. The data for number samples (small and large) are presented in the top panel, and the data for time samples (long and short) are shown in the bottom panel.

than on large-sample trials $[F(1,11)=73.81,61.01,70.07$, and 85.55].

The data for trials in which time samples were presented are shown in the bottom of the figure. For time samples, the phrase in brackets indicates the anticipated numerical coding if the pigeons coded the time samples in terms of the number of flashes at the ends of a sequence. In the last $2 \mathrm{sec}$ of each time sample, the long sample contained one light flash, whereas the short sample contained four light flashes. For time samples, a chooselong (functionally a choose-small) bias was observed. Accuracy dropped much more on short-sample trials than on long-sample trials as the delay interval increased.
For the time samples, significant effects of sample type $[F(1,11)=13.72]$ and delay interval $[F(3,44)=199.13]$ and a sample type $\times$ delay interval interaction $[F(3,44)=$ 13.03] were obtained. At the 0 -sec delay, accuracy was significantly higher on short-sample trials than on longsample trials $[F(1,11)=21.62]$. However, at all delays greater than $0 \mathrm{sec}$, accuracy was higher on the longsample trials than on the short-sample trials $[F(1,11)=$ $34.27,15.11,11.05$, and 7.89].

These results replicate those of Roberts et al. (1995) and demonstrate that their findings can be extended to a within-subjects design. The number condition produced a clear choose-small bias at all delays greater than $0 \mathrm{sec}$, 
suggesting that the pulse counts for the large sample had deteriorated over the delay interval, causing the pulse count for the large sample to be closer to the reference memory pulse count for the small sample at a 0 -sec delay. In addition, as was predicted, the time condition produced a strong choose-long bias at all delays greater than $0 \mathrm{sec}$. This is consistent with Roberts et al.'s (1995) finding, and it indicates that the pigeons were probably counting the number of flashes in the last couple of seconds of the time samples. Hence, the difference in accuracy for time samples at delays greater than $0 \mathrm{sec}$ is actually another manifestation of a choose-small bias when memory for number is tested. Overall, the findings of Experiment 1 are consistent with the idea that the processing of time and number is accomplished by the same internal clock mechanism.

\section{EXPERIMENT 2}

The second experiment of this study had two objectives. The first was to strengthen the contention that the pigeons were counting the number of flashes that occurred in the last couple of seconds of a time sample. The second objective was to confirm that in the absence of any number sample's being presented, pigeons would show a bias to respond to the small-sample comparison alternative. To accomplish these objectives, three different kinds of test sessions (no sample, number different, and number similar) were administered. During no-sample test sessions, intermittent trials occurred in which either the number or the time sample was omitted and only the comparison stimuli were presented. Previous research has demonstrated that in the absence of the presentation of an event duration sample, pigeons demonstrate a bias to respond to the comparison stimulus correct for the short-duration sample (Gaitan \& Wixted, 2000; Grant \& Spetch, 1991; Spetch \& Grant, 1993; Spetch \& Wilkie, 1983). An apparently similar bias on trials involving the absence of a number sample has been reported by Fetterman and MacEwen (1989). However, in their study the FR sample response requirement was confounded with the time taken to complete the requirement. Consequently, their choose-small effect could actually have been a choose-short effect. In the present study, presentation of the number comparison stimuli without a prior number sample would be expected to produce a bias to respond to the comparison stimulus correct for the small-number sample. In addition, because pigeons showed evidence of counting the number of light flashes during time samples in Roberts et al. (1995) and in Experiment 1 of the present study, one would expect a choose-long bias (functionally, a choose-small bias) when a time sample is omitted in the present experiment.

Additional evidence that the pigeons were counting light flashes at the end of time samples in the present study was obtained by comparing accuracy on trials in which comparison stimuli associated with number and time were mixed (i.e., a choice between a color and a line com- parison). Number-different sessions involved intermittent test trials in which pigeons were required to choose between the short (e.g., red) and the small (e.g., vertical) associated comparisons following the short and the small sample or between the long (e.g., green) and the large (e.g., horizontal) associated comparisons following the long and the large samples. Except for the novelty of being presented with a choice between a color and a line, the birds were expected to have little trouble responding accurately following either time or number samples on these trials. On trials involving presentation of the short sample, a response to the comparison stimulus associated with short samples is, in fact, a large response, and it should be selected, rather than the small response, on short versus small test trials. On trials involving the presentation of the long sample, a response to the comparison stimulus associated with long samples is really a small response, and it should be selected, rather than the large response, on long versus large test trials. Hence, the number-different test sessions were designed to ask the birds to distinguish between large (short) versus small and small (long) versus large. In contrast, numbersimilar sessions involved intermittent test trials in which the comparison stimulus pair offered a choice between the short (e.g., red) and the large (e.g., horizontal) comparisons following the short or the large sample and between the long (e.g., green) and the small (e.g., vertical) comparisons following the long or the small sample. The birds were expected to have much greater difficulty responding accurately following number and time samples on number-similar test trials, because these trials were designed to ask the birds to distinguish large (short) versus large and small (long) versus small.

\section{Method}

Subjects and Apparatus. The subjects and apparatus used in Experiment 1 were also used in Experiment 2.

Procedure. Owing to a 3-week interval between Experiments 1 and 2, the pigeons were given five sessions of the baseline training as described in Experiment 1, to ensure stable performance at the 0 -sec delay before proceeding with testing.

The testing conducted in Experiment 2 consisted of three different types of test sessions: no-sample tests, number-different tests, and number-similar tests. The six different orders of these 3 test sessions were administered to each pigeon, for a total of 18 test sessions. Each type of test session occurred once within each 3-session block. Each test session consisted of 80 regular trials, in which the samples and comparison stimuli were the same as those presented during baseline training, and 80 test trials. A correction procedure was in effect only for the regular trials. Responses on all the test trials were randomly followed by 4 -sec access to mixed grain, with a probability of .50. For no-sample sessions, the 80 test trials involved the omission of the number or the time sample and the presentation of either the number comparisons or the time comparisons immediately after the intertrial interval. For number-different sessions, the 80 test trials involved the presentation of the number sample or the time sample, followed either by a choice between the short and the small associated comparisons if the sample had been short or small or by a choice between the long and the large associated comparisons if the sample had been long or large. For numbersimilar sessions, the 80 test trials involved the presentation of the number sample or the time sample, followed either by a choice be- 

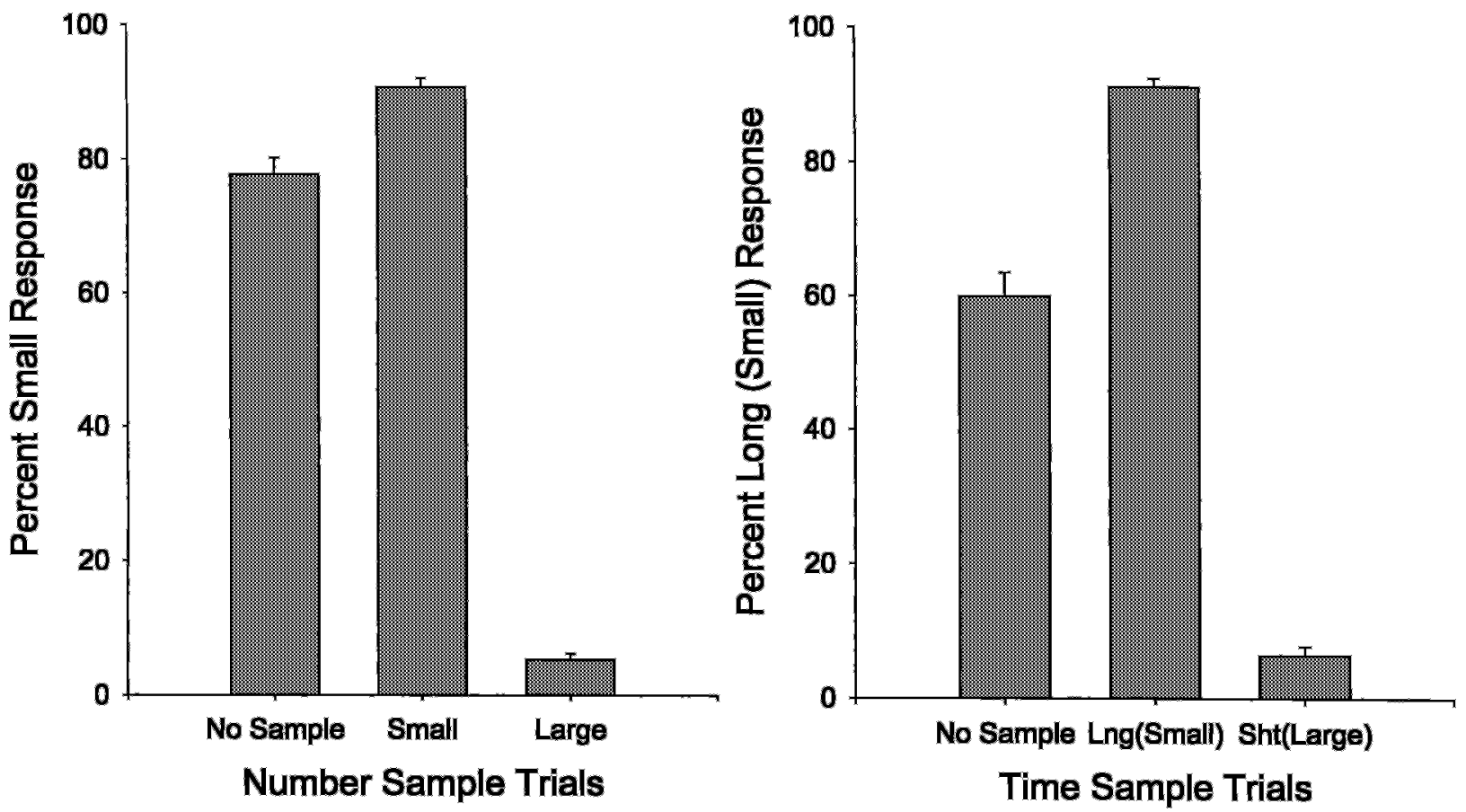

Figure 2. The mean percentage of correct choices of the comparison stimulus for the small sample on number sample trials in which no sample, the small sample, or the large sample was presented (left panel). The mean percentage of correct choices of the comparison stimulus for the long (small) sample on time sample trials in which no sample, the long (small) sample, or the short (large) sample was presented (right panel).

tween the short and the large associated comparisons if the sample had been short or large or by a choice between the long and the small associated comparisons if the sample had been long or small.

\section{Results and Discussion}

The data from sessions involving the no-sample test trials are shown in Figure 2. The data in the left panel show the mean percentage of small responses on no-sample trials, as well as on regular trials in which the small and the large samples were presented. The small comparison alternative was selected very frequently following the small sample and very infrequently following the large sample. On no-sample test trials, the small comparison alternative was selected approximately $78 \%$ of the time. The data in the right panel show the mean percentage of long responses on no-sample test trials, as well as on regular trials in which the long and the short samples were presented. Note that if the birds were coding time samples on the basis of the number of events in the last $2 \mathrm{sec}$ of each sample, the choose-long response would more appropriately be referred to as a choose-small response. The long (small) comparison alternative was selected very frequently following the long (small) sample and very infrequently following the short (large) sample. On no-sample test trials, the long (small) comparison alternative was selected approximately $60 \%$ of the time. Although the choose-small response bias obtained on nosample test trials was more evident for the number condition than for the time condition, the nature of the bias was similar. For no-sample trials in which number comparisons were presented, a single-sample $t$ test confirmed that the percentage of small responses was significantly greater than chance $[t(11)=10.98]$. In addition, for no-sample trials in which time comparisons were presented, the percentage of long (small) responses was significantly greater than chance $[t(11)=2.84]$. Thus, when no sample was presented, the birds tended to select the comparison stimulus that was correct for the sample for which they had coded the smallest number of flashes.

The results of no-sample testing are consistent with expectations derived from the mode control model. When the number comparison stimuli were presented in the absence of a sample, the pigeons showed a clear bias to choose the comparison stimulus corresponding to the small sample. This is the result predicted by the model, since no sample should more closely resemble the small sample than the large sample with respect to pulse count. When the time comparisons were presented in the absence of a sample, the pigeons showed a bias to peck the comparison stimulus that was correct for the long sample. A bias to choose long would be expected if the pigeons were in fact counting the number of flashes in the last couple of seconds of the sample. A pulse count of 0 on no-sample trials should more closely resemble the reference memory pulse count for the long (small) sample than that for the short (large) sample.

The data from sessions involving the number-different test trials are shown in the top panel of Figure 3. These data show that the pigeons continued to respond with high accuracy on test trials in which they were required 

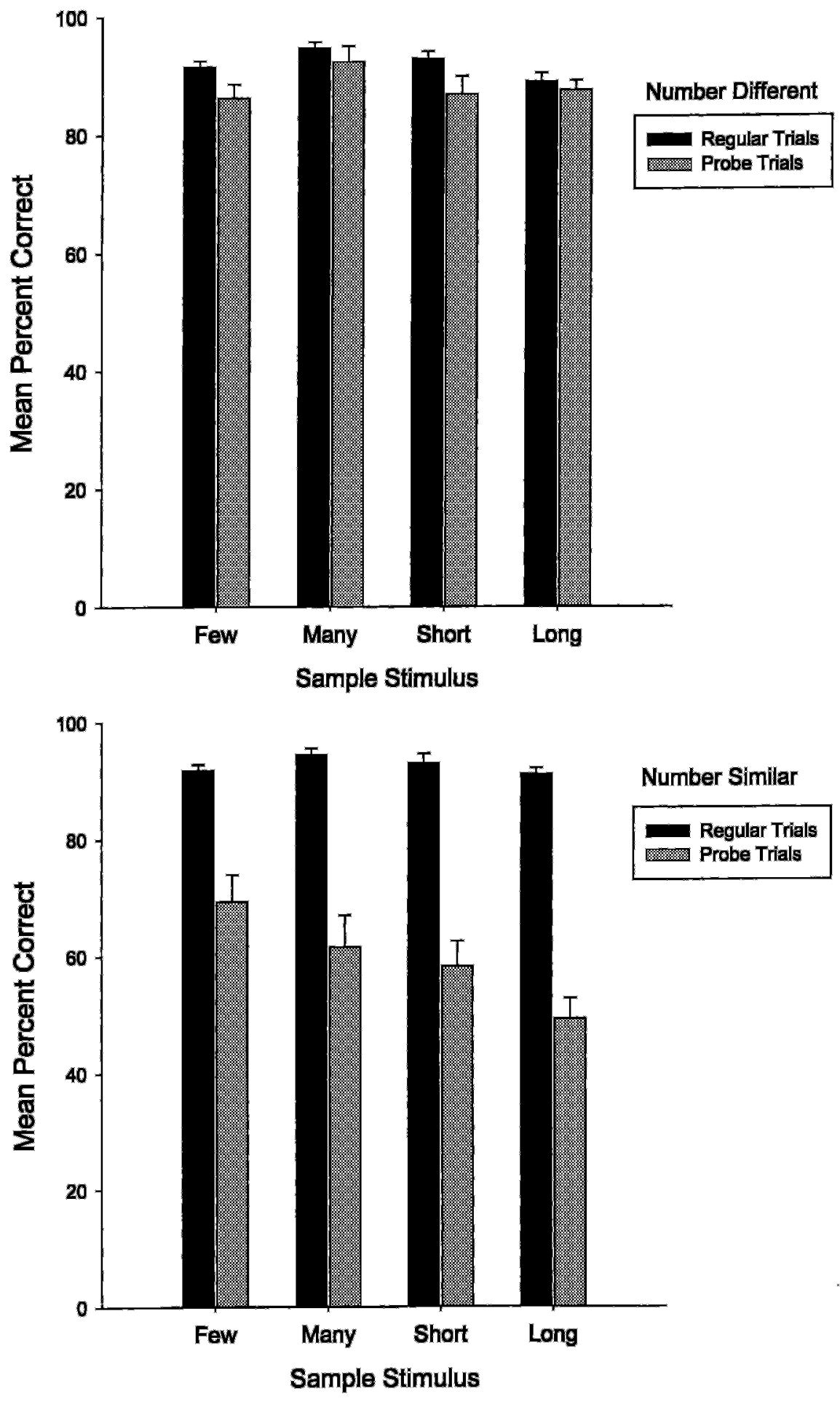

Figure 3. The mean percentage of correct responding during number-different (top panel) and number-similar (bottom panel) test sessions as a function of the sample stimulus: small, large, long (small), or short (large). On regular trials, either two color or two line comparison stimuli were presented, whereas on probe trials, a mix of one color and one line comparison was presented. Number-different probe trials required a discrimination between comparisons that were associated with samples having a large difference in number. Numbersimilar probe trials required a discrimination between comparisons that were associated with samples having very little or no difference in number. 
to distinguish small from short and large from long. The data from sessions involving the number-similar test trials are shown in the bottom panel of Figure 3. These data show a great deal of disruption when the pigeons were required to distinguish small from long and large from short. An analysis of variance indicated that there was no difference in accuracy on regular trials during numberdifferent and number-similar test sessions $[F(1,11)=$ 1.11]. However, on probe trials, accuracy was significantly lower during number-similar sessions than during numberdifferent sessions $[F(1,11)=136.43]$.

These findings are consistent with the idea that the pigeons were counting the number of flashes in the last couple of seconds of each event sequence. The last couple of seconds of the small sample and the short sample consisted of one flash and four flashes, respectively. As was shown during number-different test sessions, the pigeons had relatively little difficulty in distinguishing between small and short samples. Similarly, the last couple of seconds of the large and the long sample sequences contained four flashes and one flash, respectively. During number-different test sessions, the pigeons showed little difficulty in distinguishingbetween large and long (small) samples. On the other hand, for both the short and the large samples, four flashes occurred in the last couple of seconds of the sequences, whereas in both the long and the small samples, one flash occurred in the last couple of seconds. As a result, during number-similar test sessions, the pigeons had much greater difficulty in distinguishing between short (large) and large and between long (small) and small. These findings are in agreement with Roberts et al. (1995), and they indicate that the pigeons were counting the number of flashes in the last couple of seconds of the time samples.

\section{EXPERIMENT 3}

Previous research has demonstrated that when memory for the duration of a continuous event is assessed, the nature of the errors that occur is a function of the relationship between the baseline training delay and the test delay (Spetch, 1987; Spetch \& Rusak, 1989, 1992). At test delays longer than the baseline, choose-short errors are prevalent, whereas at test delays shorter than the baseline, choose-long errors are prevalent. The occurrence of the choose-long effect is a theoretically significant finding. It is obviously predicted by a model of event duration memory based on analogical coding and subjective shortening. However, the choose-long effect cannot be as easily accommodated by a number of alternative explanations of the choose-short effect, including an asymmetrical coding model (see Gaitan \& Wixted, 2000; Grant, Spetch, \& Kelly, 1997) and the confusion hypothesis (see Sherburne et al., 1998).

Given the theoretical importance of the choose-long effect, in Experiment 3, we attempted to demonstrate that when memory for number is tested, the nature of the errors that occur is also a function of the relationship between the baseline training delay and the test delay. All the birds were retrained with a 5-sec delay. When accuracy stabilized, testing was conducted at delays shorter than and longer than the 5-sec baseline delay. On the basis of the assumption that time and number are processed by the same mechanism, it was predicted that on trials involving number samples choose-small errors would occur at delays greater than the 5-sec baseline delay, whereas choose-large errors would occur at delays shorter than the 5-sec baseline delay. Because the time samples in the present study were not processed in terms of duration, but rather in terms of number, the data from the time sample trials actually provide additional evidence that errors in memory for number depend on the baselinetraining-test delay relationship. In the case of time samples, the short sample is functionally a large sample, and the long sample is functionally a small sample. Consequently, on trials involving time samples, choose-long errors (functionally, choose-small) should occur at delays greater than the 5-sec baseline delay, whereas chooseshort errors (functionally, choose-large) should occur at delays shorter than the 5 -sec baseline delay.

\section{Method}

Subjects and Apparatus. The subjects and apparatus used in Experiments 1 and 2 were also used in Experiment 3.

Procedure. The pigeons received 20 sessions of training with a 5-sec delay. Each session consisted of 160 trials. This training was identical to that used in Experiment 1, except that instead of a 0-sec delay between the end of the sample stimulus and the presentation of the comparison stimuli, a 5-sec delay was used. Over the last 3 sessions of 5-sec delay training, accuracy was at or above $75 \%$ correct. On the last day of training, the birds averaged $79.4 \%$ correct on the number trials and $79.5 \%$ correct on the time trials.

Once training was complete, two sets of delay testing sessions (15 sessions each) were conducted. The test sessions were similar to those in Experiment 1, except that $5 \mathrm{sec}$ was now the baseline delay, instead of $0 \mathrm{sec}$. The first set of delay tests involved delay intervals of $0,2.5,5.0,7.5$, and $10 \mathrm{sec}$. There were 4 trials each of small, large, long, and short sequences at each of 0-, 2.5-, 7.5-, and 10 -sec delay trials, whereas 24 small, 24 large, 24 long, and 24 short sequences were presented with a 5-sec delay. The second set of delay tests involved delay intervals of $0,5.0,10.0,15.0$, and $20.0 \mathrm{sec}$. There were four trials each of small, large, long, and short sequences at each of 0-, 10.0-, 15.0-, and 20-sec delay trials, whereas 24 small, 24 large, 24 long, and 24 short sequences were presented with a 5-sec delay.

\section{Results and Discussion}

The mean percentage of correct responding during the first set of delay testing sessions is shown in Figure 4. The data for trials in which number samples were presented are shown in the top of the figure, and the data for trials in which time samples were presented are shown in the bottom of the figure. For number samples, at the baseline delay of $5 \mathrm{sec}$, accuracy was equivalent on trials in which the small and the large samples were presented. However, at delays shorter than the baseline value of $5 \mathrm{sec}$, accuracy was greater on trials in which the large sample had been presented (a choose-large effect), whereas at delays longer than $5 \mathrm{sec}$, accuracy was slightly greater on trials in which the small sample had been presented. An analysis of variance of the number data indicated a 

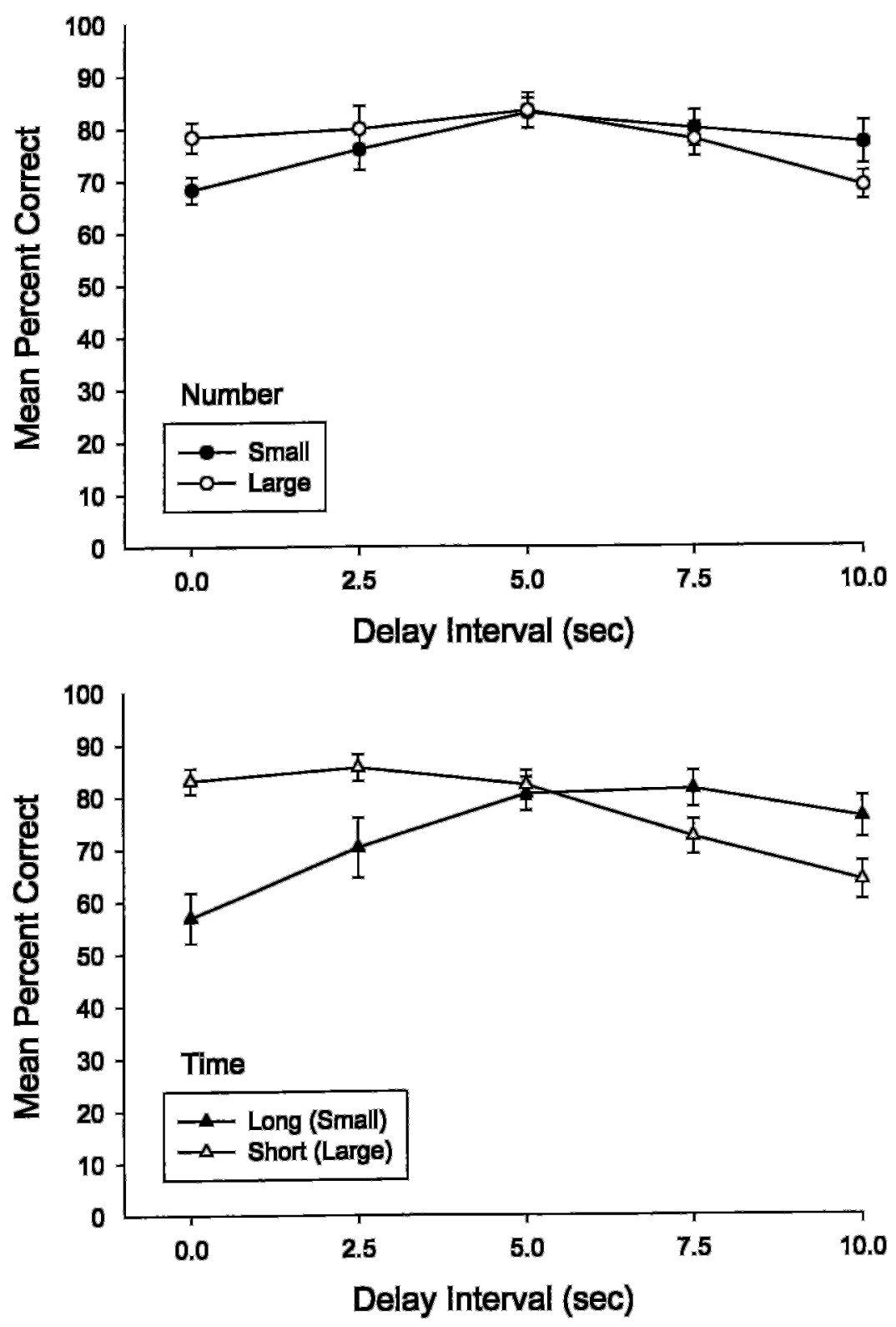

Figure 4. The mean percentage of correct responding during the first set of delay test sessions in Experiment 3. The data for number samples (small and large) are presented in the top panel, and the data for time samples (short and long) are shown in the bottom panel.

significant effect of delay $[F(4,44)=8.51]$, as well as a significant sample $\times$ delay interaction $[F(4,44)=3.15]$. A simple main effect analysis indicated that at the 5-sec baseline, accuracy was equivalent on small- and largesample trials $(F<1)$. However, at the 0 -sec delay, accuracy was significantly greater on trials in which the large sample had been presented than on trials in which the small sample had been presented $[F(1,11)=5.91]$. At the 2.5-sec delay and at delays greater than the baseline delay of $5 \mathrm{sec}$, there was no statistically significant difference in accuracy on small- and large-sample trials.
For time samples, at the baseline delay of $5 \mathrm{sec}$, accuracy was equivalent on trials in which the short and the long samples had been presented. However, at delays shorter than the baseline value of $5 \mathrm{sec}$, accuracy was markedly greater on trials in which the short sample (functionally, a large sample) had been presented. Because the pigeons were counting events during time samples, this is actually a strong choose-large effect. At delays longer than $5 \mathrm{sec}$, accuracy was greater on trials in which the long sample (functionally, a small sample) had been presented. This is actually a choose-small effect. An analysis 
of variance of the time data indicated a significant effect of delay $[F(4,44)=11.57]$, as well as a significant sample type $\times$ delay interaction $[F(4,44)=22.96]$. A simple main effect analysis indicated that at the 5-sec baseline, accuracy was equivalent on short- and long-sample trials $(F<1)$. However, at both the 0 -sec and the 2.5 -sec delay intervals, accuracy was significantly greater on trials in which the short sample (functionally, the large sample) had been presented than on trials in which the long sample (functionally, the small sample) had been presented $[F \mathrm{~s}(1,11)=34.30$ and 10.49 , respectively $]$. In con- trast, at delays greater than the baseline delay of $5 \mathrm{sec}$, accuracy was significantly greater on trials in which the long (small) sample had been presented than on trials in which the short (large) sample had been presented $\left[F_{\mathrm{S}}(1,11)=7.38\right.$ and 5.58 , respectively $]$.

The mean percentage of correct responding during the second set of delay testing sessions is shown in Figure 5. The data for trials in which number samples were presented are again shown in the top of the figure, and the data for trials in which time samples were presented are shown in the bottom of the figure. For number samples,
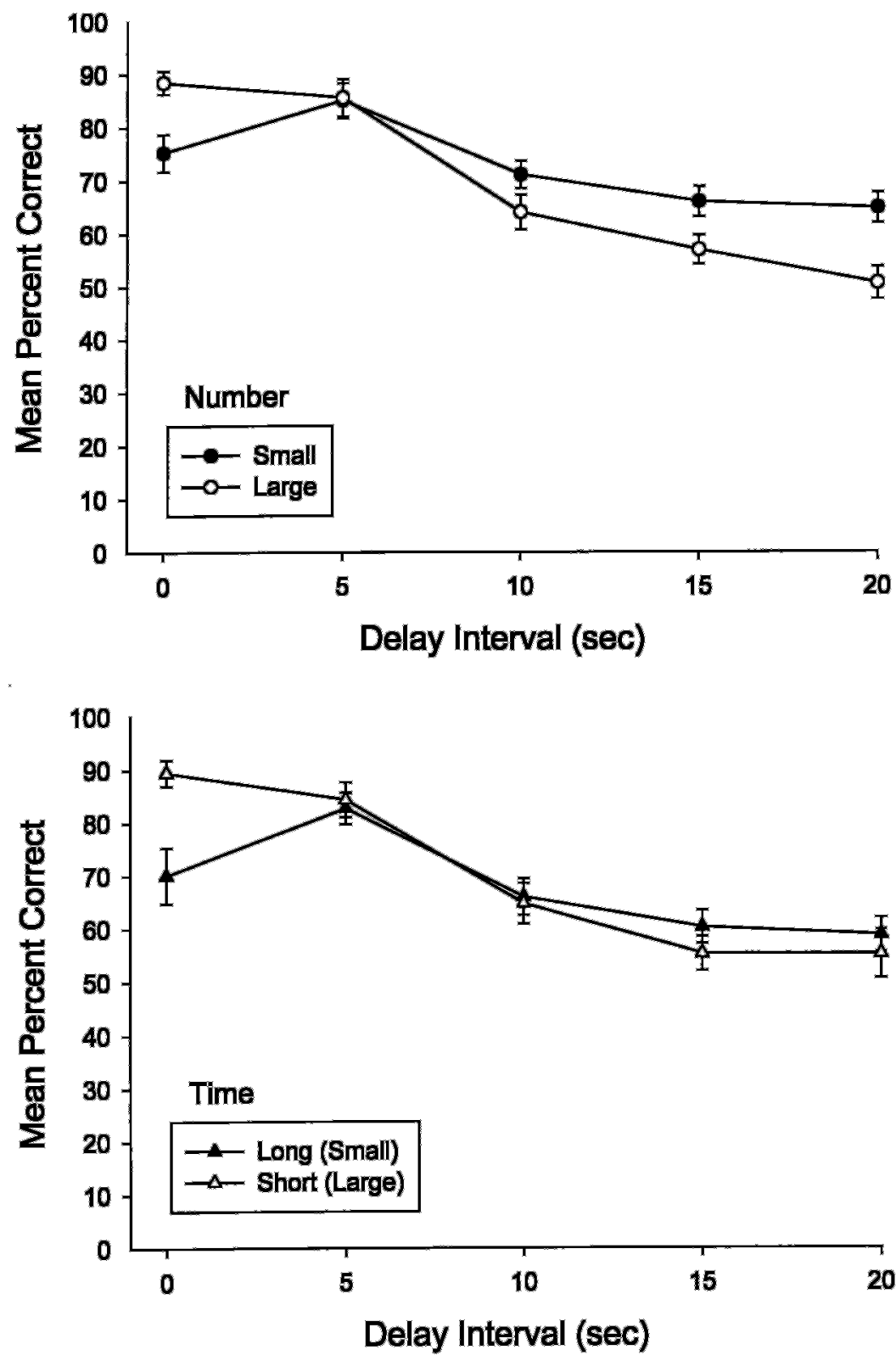

Figure 5. The mean percentage of correct responding during the second set of delay test sessions in Experiment 3. The data for number samples (small and large) are presented in the top panel, and the data for time samples (long and short) are shown in the bottom panel. 
at the baseline delay of $5 \mathrm{sec}$, accuracy was again equivalent on trials in which the small and the large samples were presented. At the 0 -sec delay, a choose-large effect was evident, whereas at delays longer than the 5-sec baseline, a choose-small effect occurred. An analysis of variance of the number data indicated a significant effect of delay $[F(4,44)=51.49]$, as well as a significant sample type $\times$ delay interaction $[F(4,44)=10.30]$. A simple main effect analysis indicated that, at the 5-sec baseline, accuracy was equivalent on small- and largesample trials $(F<1)$. However, at the 0 -sec delay, accuracy was significantly greater on trials in which the large sample had been presented than on trials in which the small sample had been presented $[F(1,11)=14.59]$. At delays greater than the baseline delay of $5 \mathrm{sec}$, accuracy was significantly greater on trials in which the small sample had been presented than on trials in which the large sample had been presented, but only at the 20 -sec delay $[F(1,11)=7.11]$.

For time samples, because the short sample was functionally a large sample, at the 0 -sec delay, a choose-large bias was evident. However, at the baseline delay of $5 \mathrm{sec}$ and longer, accuracy was equivalent on short- and longsample trials. An analysis of variance of the time data indicated a significant effect of delay $[F(4,44)=43.40]$, as well as a significant sample type $X$ delay interaction $[F(4,44)=5.18]$. A simple main effect analysis indicated that, at the 5-sec baseline, accuracy was equivalent on short- and long-sample trials $(F<1)$. However, at the 0 -sec delay, accuracy was significantly greater on trials in which the short sample (functionally, the large sample) had been presented than on trials in which the long sample had been presented $[F(1,11)=13.52]$. At delays greater than the baseline delay of $5 \mathrm{sec}$, there was no difference in accuracy between short- and long-sample trials (all $F_{\mathrm{s}}<1$ ).

The results from Experiment 3 are generally consistent with predictions of the mode control model of timing and counting. If the pulse counts in working memory are lost over a delay period, then when trained with a constant 5-sec delay, the pulse counts stored in reference memory would be smaller than they would be if no delay was used during training. Therefore, when the pigeons were tested at delays shorter than the 5-sec delay, the pulse counts in working memory would be larger than those that had been stored in reference memory. Consequently, the mode control model predicts the occurrence of a choose-large bias in the number condition of the present study. The choose-short bias observed at delays shorter than the baseline in the time condition is actually another instance of a choose-large bias, because the time samples were coded numerically. In both the first and the second sets of delay tests, the expected choose-large bias was obtained for numerical samples, as well as for numerically coded time samples. The choose-small bias in the number condition was obtained at the test delay that differed maximally from the 5-sec baseline delay (i.e., the 20-sec delay). The choose-small bias for numerically coded time samples was also obtained at test delays greater than the 5-sec baseline delay, but only in the first set of delay tests.

\section{GENERAL DISCUSSION}

The results of Experiment 1 clearly show a choosesmall effect when the sample stimuli varied in number but were equal in time. At increasing retention intervals, the large curve showed a steep drop in accuracy, whereas the small curve showed little drop in accuracy. These findings are consistent with previous results (Fetterman, 2000; Roberts et al.,1995), and they add support for the mode control model, which predicts that if there is a choose-short effect for time samples (consisting of a continuous signal), there should also be a choose-small effect for number samples. Both the choose-short and the choose-small effects can be explained by a deterioration of the pulse counts in working memory over a delay interval.

The choose-long effect for time samples (consisting of a sequence of events), which also replicates the results of Roberts et al. (1995), is really a choose-small effect. The results from Experiment 2 supported this by showing that the pigeons were highly accurate at discriminating short from small and long from large but showed disruption when required to discriminate long from small and short from large. This disruption was due to the fact that the long and small samples and the short and large samples had a similar number of events in the last couple of seconds of each sequence. The finding that pigeons count event sequences that vary in time but are constant in number, both when presented these sequences in isolation (Roberts et al., 1995) and when presented these sequences with those that vary in number but are constant in time, is an important exception to previous findings that suggest that time is a more salient dimension than number for both rats (Breukelaar \& Dalrymple-Alford, 1998, 1999; Ross \& Santi, 2000; Santi, Coppa, \& Ross, 2001) and pigeons (Roberts \& Boisvert, 1998).

Breukelaar and Dalrymple-Alford (1998) have hypothesized that superior temporal processing occurs as a result of temporal information's being considered before numerical information at the comparator phase of the internal clock model. As a result, when both temporal and numerical information are available, the temporal information will control responding, and animals become less proficient at responding on the basis of number. The present results and those of Roberts et al. (1995) for time samples are another example of the important role of stimulus factors in time and number processing. Temporal processing and/or temporal memory have been shown to be affected by stimulus modality (Meck, 1984; Roberts, Cheng, \& Cohen, 1989; Santi, Stanford, \& Coyle, 1998; Stubbs, Dreyfus, \& Fetterman, 1984), stimulus intensity (Kraemer, Brown, \& Randall, 1995; Wilkie, 1987), duration of food access versus light duration (Spetch \& Wilkie, 1982), and empty versus filled intervals (Kraemer, Randall, \& Brown, 1997; Mantanus, 
1981; Santi, Ross, Coppa, \& Coyle, 1999). It is clear from the present results that the duration of a continuous signal and the duration of a sequence of events are not always processed in the same way. The former are typically processed in terms of time, whereas the latter are sometimes processed in terms of the number of events in the last few seconds of a sequence.

Experiment 3 convincingly demonstrated that a chooselarge bias for numerically coded samples occurs at test delays that are shorter than the baseline training delay. This was obtained for number samples as well as for number-coded time samples in both sets of delay tests. The occurrence of the choose-large effect is a theoretically significant finding. It is predicted by the mode control model of timing and counting, in which samples are analogically coded and the resulting working memory codes exhibit subjective shortening. However, chooselarge and choose-long effects cannot be as easily accommodated by a number of alternative explanations of choose-small and choose-short effects, including an asymmetrical coding model (see Gaitan \& Wixted, 2000; Grant et al., 1997) and the confusion hypothesis (see Sherburne et al., 1998).

Delay testing during Experiment 3 replicated the choose-small effect for number samples, but only for the second set of delay tests, which included delays that where longer than those in the first set. Whereas it is understandable that a choose-small effect would be more evident at test delays that are maximally different from the baseline training delay, it is not as clear why this would not be equally true for the number-coded time samples. For number-coded time samples, a significant choosesmall effect occurred during the first set of delay tests, but not during the second set of delay tests. It is difficult to explain the absence of a choose-small effect for numbercoded time samples in the second delay test.

Our accounting of the present findings is similar to that proposed by Roberts et al. (1995) in that we have argued that the number of events is the variable controlling choice. However, as a reviewer of the present manuscript noted, it is possible that the pigeons, in both studies, used the rate of light flashes as the basis for discriminating between samples. Although the present data cannot rule out such a possibility, it is difficult to understand exactly how testing at a delay shorter than the training delay produces a choose-fast effect. The coded representations of fast and slow flash rates would have to undergo some form of subjective truncation analogous to the subjective shortening of time or the subjective shrinking of number. In addition, it is clear that pigeons are perfectly capable of discriminating flash sequences on the basis of number independently of rate. Roberts and Mitchell (1994) trained pigeons to discriminate between sequences of 200-msec light flashes presented at the rate of $1 / \mathrm{sec}$ that lasted either 2 or $8 \mathrm{sec}$. A peck left was correct for $2 \mathrm{f} / 2 \mathrm{sec}$, and a peck right was correct for $8 \mathrm{f} / 8 \mathrm{sec}$ flashes. In their study, rate could not be used to discriminate the two sequences; yet, pigeons showed independent and equivalent stimulus control by both time and number. Of course, it is possible that pigeons rely on time or number only when rate cannot serve as the controlling dimension. Additional empirical work is needed to definitively rule out a rate account of the present findings and those of Roberts et al. (1995).

The main objective of the present study was to provide additional empirical support for conceptualizing memory for number and time in terms of a common mechanism. Experiment 1 demonstrated that the choose-small bias for number samples and the choose-long (functionally, choose-small) bias for time samples reported by Roberts et al. (1995) can be obtained in a within-subjects design. Experiment 2 provided a clearer demonstration than did Fetterman and MacEwen (1989) of a choosesmall bias on trials involving the absence of number samples or the absence of number-coded time samples. It also provided additional data that were consistent with the contention that the pigeons coded time samples in terms of the number of flashes occurring in the last few seconds of a sequence. In Experiment 3, the birds received baseline training with a 5-sec delay and were subsequently tested at shorter and longer delays. A choose-large bias reliably occurred at the test delays that were shorter than the baseline training delay, whereas a choose-small bias was again observed at delays longer than the baseline delay for both number samples and number-coded time samples during one of the two sets of delay tests. These findings provide additional empirical support for the conceptualizing of memory for number and time in terms of a common mechanism.

\section{REFERENCES}

Breukelaar, J. W. C., \& Dalrymple-Alford, J. C. (1998). Timing ability and numerical competence in rats. Journal of Experimental Psychology: Animal Behavior Processes, 24, 84-97.

BreukelaAr, J. W. C., \& Dalry mple-Alford, J. C. (1999). Effects of lesions to the cerebellar vermis and hemispheres on timing and counting in rats. Behavioral Neuroscience, 113, 78-90.

Broadbent, H. A., Church, R. M., Meck, W. H., \& Rakitin, B. C. (1993). Quantitative relationships between counting and timing. In S. T. Boysen \& E. J. Capaldi (Eds.), The development of numerical competence: Animal and human models (pp. 171-187). Hillsdale, NJ: Erlbaum.

Fetterman, J. G. (1995). The psychophysics of remembered duration. Animal Learning \& Behavior, 23, 49-62.

Fetterman, J. G. (2000). Biases in pigeon working memory. Animal Learning \& Behavior, 28, 68-79.

Fetterman, J. G., \& MacEwen, D. (1989). Short-term memory for responses: The "choose-small" effect. Journal of the Experimental Analysis of Behavior, 52, 311-324.

Gaitan, S. C., \& Wixted, J. T. (2000). The role of "nothing" in memory for event duration in pigeons. Animal Learning \& Behavior, 28, 147-161.

Grant, D. S. (1993). Coding processes in pigeons. In T. R. Zentall (Ed.), Animal cognition: A tribute to Donald A. Riley (pp. 193-216). Hillsdale, NJ: Erlbaum.

Grant, D. S., \& Spetch, M. L. (1991). Pigeons' memory for event duration: Differences between choice and successive matching tasks. Learning \& Motivation, 22, 180-199.

Grant, D. S., \& Spetch, M. L. (1993). Analogical and nonanalogical coding of samples differing in duration in a choice-matching task in pigeons. Journal of Experimental Psychology: Animal Behavior Processes, 19, 15-25.

Grant, D. S., \& Spetch, M. L. (1994). The role of asymmetrical cod- 
ing of duration samples in producing the choose-short effect in pigeons. Learning \& Motivation, 25, 413-430.

Grant, D. S., Spetch, M. L., \& Kelly, R. (1997). Pigeons' coding of event duration in delayed matching-to-sample. In C. M. Bradshaw \& E. Szabadi (Eds.), Time and behaviour: Psychological and neurobehavioural analyses (pp. 217-264). Amsterdam: Elsevier.

Kelly, R., \& Spetch, M. L. (2000). Choice biases in delayed matchingto-sample duration with pigeons: Manipulations of ITI and delay illumination. Quarterly Journal of Experimental Psychology, 53B, 309-323.

Kraemer, P. J., Brown, R. W., \& Randall, C. K. (1995). Signal intensity and duration estimation in rats. Behavioural Processes, 34, 265-268.

Kraemer, P. J., Mazmanian, D. S., \& Roberts, W. A. (1985). The choose-short effect in pigeon memory for stimulus duration: Subjective shortening versus coding models. Animal Learning \& Behavior, 13, 349-354.

Kraemer,P. J., Randall, C. K., \& Brown, R. W. (1997).The influence of stimulus attributes on duration matching-to-sample in pigeons. Animal Learning \& Behavior, 25, 148-157.

Mantanus, H. (1981). Empty and filled interval discrimination by pigeons. Behaviour Analysis Letters, 1, 217-224.

Meck, W. H. (1984). Attentional bias between modalities: Effect on the internal clock, memory, and decision stages used in animal time discrimination. In J. Gibbon \& L. Allan (Eds.), Timing and time perception (Annals of the New York Academy of Sciences, Vol. 423, pp. 528541). New York: New York Academy of Sciences.

Meck, W. H., \& Church, R. M. (1983). A mode control model of counting and timing processes. Journal of Experimental Psychology: Animal Behavior Processes, 9, 320-334.

RoBerts, W. A. (1998). Principles of animal cognition. Boston: McGrawHill.

Roberts, W. A., \& Boisvert, M. J. (1998). Using the peak procedure to measure timing and counting processes in pigeons. Journal of Experimental Psychology: Animal Behavior Processes, 24, 416-430.

Roberts, W. A., Cheng, K., \& Cohen, J. (1989). Timing light and tone signals in pigeons. Journal of Experimental Psychology: Animal Behavior Processes, 15, 23-35.

Roberts, W. A., Macuda, T., \& Brodbeck, D. R. (1995). Memory for number of light flashes in the pigeon. Animal Learning \& Behavior, 23, $182-188$.

Roberts, W. A., \& Mitchell, S. (1994). Can a pigeon simultaneously process temporal and numerical information? Journal of Experimental Psychology: Animal Behavior Processes, 20, 66-78.

Ross, L., \& SANTI, A. (2000). The effects of estrogen on temporal and numerical processing in ovariectomized female rats. Psychobiology, 28, 394-405.

Santi, A., Bridson, S., \& Ducharme, M. J. (1993). Memory codes for temporal and nontemporal stimuli in many-to-one matching by pigeons. Animal Learning \& Behavior, 21, 120-130.
SAnTi, A., Coppa, R., \& Ross, L. (2001). Effects of the dopamine D2 agonist quinpirole on time and number processing in rats. Pharmacology, Biochemistry \& Behavior, 68, 147-155.

Santi, A., Ducharme, M. J., \& Bridson, S. (1992). Differential outcome expectancies and memory for temporal and nontemporal stimuli in pigeons. Learning \& Motivation, 23, 156-169.

Santi, A., Ross, L., Coppa, R., \& Coyle, J. (1999). Pigeons' memory for empty time intervals marked by visual or auditory stimuli. Animal Learning \& Behavior, 27, 190-205.

Santi, A., Stanford, L., \& Coy Le, J. (1998). Pigeons' memory for event duration: Differences between visual and auditory signals. Animal Learning \& Behavior, 26, 163-171.

Sherburne, L. M., Zentall, T. R, \& Kaiser, D. H. (1998). Timing in pigeons: The choose-short effect may result from pigeons' "confusion" between delay and intertrial intervals. Psychonomic Bulletin \& Review, 5, 516-522.

SPETCH, M. L. (1987). Systematic errors in pigeons' memory for event duration: Interaction between training and test delay. Animal Learning \& Behavior, 15, 1-5.

Spetch, M. L., \& GRANT, D. S. (1993). Pigeons' memory for event duration in choice and successive matching-to-sample tasks. Learning \& Motivation, 24, 156-174.

SPETCH, M. L., \& RuSAK, B. (1989). Pigeons' memory for event duration: Intertrial interval and delay effects. Animal Learning \& Behavior, 17, 147-156.

Spetch, M. L., \& Rusak, B. (1992). Temporal context effects in pigeons' memory for event duration. Learning \& Motivation, 23, 117 144.

Spetch, M. L., \& Wilkie, D. M. (1982). A systematic bias in pigeons' memory for food and light durations. Behavior Analysis Letters, 2, 267-274.

SPetch, M. L., \& Wilkie, D. M. (1983). Subjective shortening: A model of pigeons' memory for event durations. Journal of Experimental Psychology: Animal Behavior Processes, 9, 14-30.

STADDON, J. E. R. \& Higa, J. J. (1999). Time and memory: Towards a pacemaker-free theory of interval timing. Journal of the Experimental Analysis of Behavior, 71, 215-251.

Stubis, D. A., Dreyfus, L. R., \& Fetterman, J. G. (1984). The perception of temporal events. In J. Gibbon \& L. Allan (Eds.), Timing and time perception (Annals of the New York Academy of Sciences, Vol. 423, pp. 30-42). New York: New York Academy of Sciences.

WILKIE, D. M. (1987). Stimulus intensity affects pigeons' timing behavior: Implications for an internal clock model. Animal Learning \& Behavior, 15, 35-39.

(Manuscript received January 31, 2001; revision accepted for publication April 16, 2001.) 